\title{
An Approximate Solution of the Time-Fractional Fisher Equation with Small Delay by Residual Power Series Method
}

\author{
Ali Demir (D), ${ }^{1}$ Mine Aylin Bayrak, ${ }^{1}$ and Ebru Ozbilge $\mathbb{D}^{2}$ \\ ${ }^{1}$ Department of Mathematics, University of Kocaeli, Kocaeli, Turkey \\ ${ }^{2}$ American University of the Middle East, Department of Mathematics and Statistics, Egaila, Kuwait \\ Correspondence should be addressed to Ali Demir; ademir@kocaeli.edu.tr
}

Received 22 May 2018; Revised 13 July 2018; Accepted 1 August 2018; Published 26 September 2018

Academic Editor: Isabel S. Jesus

Copyright (c) 2018 Ali Demir et al. This is an open access article distributed under the Creative Commons Attribution License, which permits unrestricted use, distribution, and reproduction in any medium, provided the original work is properly cited.

\begin{abstract}
An analytical solution of the time-fractional Fisher equation with small delay is established by means of residual the residual power series method (RPSM) where the fractional derivative is taken in the Caputo sense. Taking advantage of small delay, the timefractional Fisher equation is expanded in powers series of delay term $\epsilon$. By using RPSM analytical solution of time-fractional of Fisher equation is constructed. The final results and graphical consequences illustrate that the proposed method in this study is very efficient, effective, and reliable for the solution of the time-fractional Fisher equation with small delay.
\end{abstract}

\section{Introduction}

In last few decades, fractional calculus growing considerable interest is used in bioengineering, thermodynamics, viscoelasticity, control theory, aerodynamics, electromagnetics, signal processing, chemistry, and finance [1-7]. Many numerical methods have been applied and analyzed for differential equations with fractional order derivative of Riemann-Liouville or Caputo sense [6-12]. Delay differential equations (DDEs) can be considered as the generalization of the ordinary differential equations which are appropriate for modelling physical systems with memory. The delay is an important fact of the physical systems such as kinetics, controllers, signal processing, and damping behavior of viscoelastic materials.

The RPSM was established as a powerful method for fuzzy differential equations [13]. It has been successfully put into practice in various fields [14-20]. The solution of problems by RPSM is obtained in the form of Maclaurin series. It is an efficient method to find out the coefficients of the series solutions. Construction of multidimensional and multiple solutions for fractional differential equations in the form of power series is an important advantage of RPSM. RPSM is effective and easy to use for solving linear and nonlinear FPDEs without linearization, perturbation, or discretization.
In this study, we extend the application of the RPSM in order to establish an approximate solution to time-fractional Fisher equation with small delay

$$
\begin{aligned}
D_{t}^{\alpha} u(x, t)= & D_{x x} u(x, t) \\
& +6 u(x, t-\epsilon)(1-u(x, t-\epsilon)), \\
& x \in R, t>0,0<\alpha \leqslant 1,
\end{aligned}
$$

subject to initial condition

$$
u(x, 0)=A_{1}(x)
$$

which was originally proposed by Fisher [20] which describes the spatial and temporal propagation of a virile gene in an infinite medium. The time-fractional Fisher equation can be readily solved by many methods [21-25]. Moreover, there are various studies on nonlocal of action of fractional differential equations including space fractional derivative [26-30]. In this article, by making use of the residual power series method (RPSM), we find series solution for the time-fractional Fisher equation with small delay. By taking advantage of the small delay $\epsilon$ we expand the term including small delay to Taylor series which reduced the problem to a perturbation problem. Applying RPSM we determine the solution of equations which are the obtained from the 
coefficients of $\epsilon$. Replacing these solutions in the Taylor series the solution of the original problem is obtained.

\section{Preliminaries}

In this section, the basic definitions and various features for fractional calculus theory are shown [6,31-35].

Definition 1. The Riemann-Liouville fractional integral of order $\alpha(\alpha \geq 0)$ is given as [14]

$$
\begin{aligned}
& J^{\alpha} f(x)=\frac{1}{\Gamma(\alpha)} \int_{0}^{x}(x-t)^{\alpha-1} f(t) d t, \quad \alpha>0, x>0, \\
& J^{0} f(x)=f(x)
\end{aligned}
$$

Definition 2. The Caputo fractional derivative with order $\alpha$ is given as $[11,12]$

$$
\begin{aligned}
D^{\alpha} f(x) & =J^{m-\alpha} D^{m} f(x) \\
& =\int_{0}^{x}(x-t)^{m-\alpha-1} \frac{d^{m}}{d t^{m}} f(t) d t, \\
& m-1<\alpha<m, x>0,
\end{aligned}
$$

where $D^{m}$ is the classic differential operator with order $m$.

By the Caputo derivative, we get

$$
\begin{aligned}
& D^{\alpha} x^{\beta}=0, \quad \beta<\alpha, \\
& D^{\alpha} x^{\beta}=\frac{\Gamma(\beta+1)}{\Gamma(\beta+1-\alpha)} x^{\beta-\alpha}, \quad \beta \geq \alpha .
\end{aligned}
$$

Definition 3. Caputo's time-fractional derivative of order $\alpha$ of $u(x, t)$ is defined as $[11,12]$

$$
\begin{aligned}
& D_{t}^{\alpha} u(x, t) \\
& = \begin{cases}\frac{1}{\Gamma(m-\alpha)} \int_{0}^{t}(t-\xi)^{m-\alpha-1} \frac{\partial^{m} u(x, \xi)}{\partial t^{m}} d \xi, & m-1<\alpha<m \\
\frac{\partial^{m} u(x, t)}{\partial t^{m}}, & \alpha=m \in N .\end{cases}
\end{aligned}
$$

Definition 4. A power series expansion of the form

$$
\begin{aligned}
\sum_{m=0}^{\infty} c_{m}\left(t-t_{0}\right)^{m \alpha}= & c_{0}+c_{1}\left(t-t_{0}\right)^{\alpha}+c_{2}\left(t-t_{0}\right)^{2 \alpha} \\
& +\cdots, \\
& 0 \leq m-1<\alpha \leq m, t \geq t_{0}
\end{aligned}
$$

is called fractional power series about $t=t_{0}[25]$.

Definition 5. A power series expansion of the form

$$
\begin{aligned}
\sum_{m=0}^{\infty} f_{m}(x)\left(t-t_{0}\right)^{m \alpha}= & f_{0}(x)+f_{1}(x)\left(t-t_{0}\right)^{\alpha} \\
& +f_{2}(x)\left(t-t_{0}\right)^{2 \alpha}+\cdots, \\
& 0 \leq m-1<\alpha \leq m, t \geq t_{0}
\end{aligned}
$$

is called multiple fractional power series about $t=t_{0}[11,12]$.
Theorem 6. Suppose that $u(x, t)$ has a multiple fractional power series representation at $t=t_{0}$ of the form

$$
u(x, t)=\sum_{m=0}^{\infty} f_{m}(x)\left(t-t_{0}\right)^{m \alpha},
$$

$$
x \in I, t_{0} \leq t \leq t_{0}+R
$$

If $D_{t}^{m \alpha} u(x, t), m=0,1,2, \ldots$, are continuous on $I \times\left(t_{0}, t_{0}+R\right)$, then $f_{m}(x)=D_{t}^{m \alpha} u\left(x, t_{0}\right) / \Gamma(m \alpha+1)$.

\section{RPSM of the Time-Fractional Fisher Equation with Small Delay}

Consider the time-fractional Fisher equation (1) with small delay. Since the delay term is very small, we replace $u(x, t-\epsilon)$ (1) by the following Taylor series expansion:

$$
u(x, t-\epsilon)=u(x, t)-\epsilon D_{t} u(x, t)+O\left(\epsilon^{2}\right),
$$

where we ignore the higher order terms, which leads to

$$
\begin{aligned}
D_{t}^{\alpha} u(x, t)= & D_{x x} u(x, t)+6 u(x, t)(1-u(x, t)) \\
& +6 \epsilon u D_{t} u-6 \epsilon(1-u) D_{t} u-6 \epsilon^{2}\left(D_{t} u\right)^{2}
\end{aligned}
$$

and assuming that $n \alpha=1$, we obtain

$$
\begin{aligned}
D_{t}^{\alpha} u(x, \mathrm{t})= & D_{x x} u(x, t)+6 u(x, t)(1-u(x, t)) \\
& +6 \epsilon u D_{t}^{n \alpha} u-6 \epsilon(1-u) D_{t}^{n \alpha} u \\
& -6 \epsilon^{2}\left(D_{t}^{n \alpha} u\right)^{2} .
\end{aligned}
$$

In this study we obtain the solution of time-fractional Fisher equation with small delay for $n=2$; hence we get the following equation:

$$
\begin{gathered}
D_{t}^{\alpha} u(x, t)-D_{x x} u(x, t)-6 u(x, t)(1-u(x, t)) \\
-6 \epsilon(2 u-1) D_{t}^{2 \alpha} u+6 \epsilon^{2}\left(D_{t}^{2 \alpha} u\right)^{2}=0 .
\end{gathered}
$$

Using (12) in (1) we reduce the problem to a perturbation problem for which we use the following series solution:

$$
u=u_{0}+\epsilon u_{1}+\epsilon^{2} u_{2}+\epsilon^{3} u_{3}+\cdots
$$

to obtain the solution of (14). Substituting (15) into (14) leads to the following equation:

$$
\begin{aligned}
& D_{t}^{\alpha}\left(u_{0}+\epsilon u_{1}+\epsilon^{2} u_{2}+\epsilon^{3} u_{3}+\cdots\right)-D_{x x}\left(u_{0}+\epsilon u_{1}\right. \\
& \left.+\epsilon^{2} u_{2}+\epsilon^{3} u_{3}+\cdots\right)-6\left(u_{0}+\epsilon u_{1}+\epsilon^{2} u_{2}+\epsilon^{3} u_{3}\right. \\
& \quad+\cdots)+6\left(u_{0}+\epsilon u_{1}+\epsilon^{2} u_{2}+\epsilon^{3} u_{3}+\cdots\right)^{2} \\
& \quad+12 \epsilon\left(u_{0}+\epsilon u_{1}+\epsilon^{2} u_{2}+\epsilon^{3} u_{3}+\cdots\right)\left(D_{t}^{2 \alpha} u_{0}\right. \\
& \left.\quad+\epsilon D_{t}^{2 \alpha} u_{1}+\epsilon^{2} D_{t}^{2 \alpha} u_{2}+\epsilon^{3} D_{t}^{2 \alpha} u_{3}+\cdots\right) \\
& \quad+6 \epsilon\left(D_{t}^{2 \alpha} u_{0}+\epsilon D_{t}^{2 \alpha} u_{1}+\epsilon^{2} D_{t}^{2 \alpha} u_{2}+\epsilon^{3} D_{t}^{2 \alpha} u_{3}\right.
\end{aligned}
$$




$$
\begin{aligned}
& +\cdots)+6 \epsilon^{2}\left(D_{t}^{2 \alpha} u_{0}+\epsilon D_{t}^{2 \alpha} u_{1}+\epsilon^{2} D_{t}^{2 \alpha} u_{2}\right. \\
& \left.+\epsilon^{3} D_{t}^{2 \alpha} u_{3}+\cdots\right)=0
\end{aligned}
$$

Hence we get the following equations:

$$
\begin{aligned}
& D_{t}^{\alpha} u_{0}-D_{x x} u_{0}-6 u_{0}+6 u_{0}^{2}=0 \\
& D_{t}^{\alpha} u_{1}-D_{x x} u_{1}-6 u_{1}+12 u_{0} u_{1}-12 u_{0} D_{t}^{2 \alpha} u_{0} \\
& \quad+6 D_{t}^{2 \alpha} u_{0}=0 \\
& D_{t}^{\alpha} u_{2}-D_{x x} u_{2}-6 u_{2}+6 u_{1}^{2}+12 u_{0} u_{2}-12 u_{0} D_{t}^{2 \alpha} u_{1} \\
& \quad-12 u_{1} D_{t}^{2 \alpha} u_{0}+6 D_{t}^{2 \alpha} u_{1}+6\left(D_{t}^{2 \alpha} u_{0}\right)^{2}=0 \\
& D_{t}^{\alpha} u_{3}-D_{x x} u_{3}-6 u_{3}+12 u_{0} u_{3}+12 u_{1} u_{2} \\
& \quad-12 u_{0} D_{t}^{2 \alpha} u_{2}-12 u_{1} D_{t}^{2 \alpha} u_{1}-12 u_{2} D_{t}^{2 \alpha} u_{0} \\
& \quad+6 D_{t}^{2 \alpha} u_{2}+12 D_{t}^{2 \alpha} u_{0} D_{t}^{2 \alpha} u_{1}=0
\end{aligned}
$$

and so on. We apply the RPSM to find out series solution for these equation subject to given initial conditions by replacing its fractional power series expansion with its truncated residual function. From each equation, a repetition formula for the calculation of coefficients is supplied, while coefficients in fractional power series expansion can be calculated by repeatedly fractional differentiation of the truncated residual function [13-20]. The RPSM propose the solutions for (18)(21) as a fractional power series about the initial point $t=0$ [13]

$$
\begin{aligned}
u_{i}(x, t)=\sum_{k=0}^{\infty} f_{i, k}(x) \frac{t^{k \alpha}}{\Gamma(k \alpha+1)}, & \\
& \quad i=0,1,2,3, x \in I, 0 \leq t<R .
\end{aligned}
$$

To obtain the numerical values from this series, let $u_{m}(x, t)$ denote the $m$-th truncated series of $u(x, t)$. That is,

$$
\begin{aligned}
u_{i, m}(x, t)=\sum_{k=0}^{m} f_{i, k}(x) & \frac{t^{k \alpha}}{\Gamma(k \alpha+1)}, \\
& i=0,1,2,3, x \in I, 0 \leq t<R .
\end{aligned}
$$

By the initial condition, the 0 th residual power series approximate solution of $u(x, t)$ can be written as follows:

$$
u_{0}(x, t)=f_{0}(x)=u(x, 0)=A_{1}(x)
$$

Equation (22) can be written as

$$
\begin{aligned}
u_{m}(x, t)=A_{1}(x) & +\sum_{k=2}^{m} A_{k}(x) \frac{t^{k \alpha}}{\Gamma(k \alpha+1)}, \\
0 & <\alpha \leq 1, x \in I, 0 \leq t, k=2,3, \ldots .
\end{aligned}
$$

Define the residual function for (18) [35]

$$
\operatorname{Res}_{0}(x, t)=D_{t}^{\alpha} u_{0}-D_{x x} u_{0}-6 u_{0}+6 u_{0}^{2}
$$

and the $m$ th residual function can be expressed as

$$
\operatorname{Res}_{m}(x, t)=D_{t}^{\alpha} u_{m}-D_{x x} u_{m}-6 u_{m}+6 u_{m}^{2}
$$

From [13-23], by making use of some results such as $\operatorname{Res}(x, t)=0$ for each $x \in I$ and $t \geq 0$ and $D_{t}^{r \alpha} \operatorname{Res}(x, 0)=$ $D_{t}^{r \alpha} \operatorname{Res}_{m}(x, 0)=0, r=0,1,2, \ldots, m$ are used to obtain the solution.

Substituting the $\mathrm{m} t h$ truncated series of $u(x, t)$ into (18), calculating the fractional derivative $D_{t}^{(m-1) \alpha}$ of $\operatorname{Res}(x, t), m=$ $1,2,3, \ldots$, at $t=0$ and solving the following obtained algebraic system

$$
D_{t}^{(m-1) \alpha} \operatorname{Res}_{m}(x, 0)=0, \quad 0<\alpha \leq 1, m=1,2,3, \ldots,
$$

the required coefficients $A_{k}(x), k=2,3, \ldots, m$ in (24) are determined.

In order to determine $A_{2}(x)$, the 1 st residual function in (26) can be written as follows:

$$
\operatorname{Res}_{0,1}(x, t)=D_{t}^{\alpha} u_{0,1}-D_{x x} u_{0,1}-6 u_{0,1}+6 u_{0,1}^{2}
$$

where $u_{0,1}(x, t)=A_{1}(x)+A_{2}(x)\left(t^{\alpha} / \Gamma(1+\alpha)\right)$. Therefore,

$$
\begin{aligned}
\operatorname{Res}_{0,1}(x, t)= & A_{2}-\left(A_{1}^{\prime \prime}+A_{2}^{\prime \prime} \frac{t^{\alpha}}{\Gamma(1+\alpha)}\right) \\
& -6\left(A_{1}+A_{2} \frac{t^{\alpha}}{\Gamma(1+\alpha)}\right) \\
& +6\left(A_{1}+A_{2} \frac{t^{\alpha}}{\Gamma(1+\alpha)}\right)^{2} .
\end{aligned}
$$

From (27), we deduce that $\operatorname{Res}_{1}(x, 0)=0$, which leads to

$$
A_{2}(x)=A_{1}^{\prime \prime}+6 A_{1}-6 A_{1}^{2} \text {. }
$$

Similarly, to obtain $A_{3}(x)$, the 2 nd residual function in (26) can be written in the following form:

$$
\operatorname{Res}_{0,2}(x, t)=D_{t}^{\alpha} u_{0,2}-D_{x x} u_{0,2}-6 u_{0,2}+6 u_{0,2}^{2}
$$

where $u_{0,2}(x, t)=A_{1}(x)+A_{2}(x)\left(t^{\alpha} / \Gamma(1+\alpha)\right)+$ $A_{3}(x)\left(t^{2 \alpha} / \Gamma(1+2 \alpha)\right)$. Therefore,

$$
\begin{aligned}
\operatorname{Res}_{0,2}(x, t) & \\
= & \left(A_{2}(x)+A_{3}(x) \frac{t^{\alpha}}{\Gamma(1+\alpha)}\right) \\
& -\left(A_{1}^{\prime \prime}+A_{2}^{\prime \prime} \frac{t^{\alpha}}{\Gamma(1+\alpha)}+A_{3}^{\prime \prime} \frac{t^{2 \alpha}}{\Gamma(1+2 \alpha)}\right) \\
& -6\left(A_{1}+A_{2} \frac{t^{\alpha}}{\Gamma(1+\alpha)}+A_{3} \frac{t^{2 \alpha}}{\Gamma(1+2 \alpha)}\right) \\
& +6\left(A_{1}+A_{2} \frac{t^{\alpha}}{\Gamma(1+\alpha)}+A_{3} \frac{t^{2 \alpha}}{\Gamma(1+2 \alpha)}\right)^{2} .
\end{aligned}
$$


The operator $D_{t}^{\alpha}$ is applied on both sides of (32) as follows:

$$
\begin{aligned}
& D_{t}^{\alpha} \operatorname{Res}_{0,2}(x, t)=A_{3}-\left(A_{2}^{\prime \prime}+A_{3}^{\prime \prime} \frac{t^{\alpha}}{\Gamma(1+\alpha)}\right) \\
& -6\left(A_{2}+A_{3} \frac{t^{\alpha}}{\Gamma(1+\alpha)}\right) \\
& +6\left(A_{2}+A_{3} \frac{t^{\alpha}}{\Gamma(1+\alpha)}\right) \\
& \cdot\left(A_{1}+A_{2} \frac{t^{\alpha}}{\Gamma(1+\alpha)}+A_{3} \frac{t^{2 \alpha}}{\Gamma(1+2 \alpha)}\right) \\
& +6\left(A_{1}+A_{2} \frac{t^{\alpha}}{\Gamma(1+\alpha)}+A_{3} \frac{t^{2 \alpha}}{\Gamma(1+2 \alpha)}\right) \\
& .\left(A_{2}+A_{3} \frac{t^{\alpha}}{\Gamma(1+\alpha)}\right)
\end{aligned}
$$

From (27) and (33),

$$
A_{3}(x)=A_{2}^{\prime \prime}+6 A_{2}-12 A_{1} A_{2} .
$$

The same manner is repeated as above; the following recurrence results is obtained

$$
A_{4}(x)=A_{3}^{\prime \prime}+6 A_{3}-12 A_{1} A_{3}-12 A_{2}^{2}
$$

and so on.

Thus, we have

$$
\begin{aligned}
u_{0}= & A_{1}+\left(A_{1}^{\prime \prime}+6 A_{1}-6 A_{1}^{2}\right) \frac{t^{\alpha}}{\Gamma(1+\alpha)} \\
& +\left(A_{2}^{\prime \prime}+6 A_{2}-12 A_{1} A_{2}\right) \frac{t^{2 \alpha}}{\Gamma(1+2 \alpha)} \\
& +\left(A_{3}^{\prime \prime}+6 A_{3}-12 A_{1} A_{3}-12 A_{2}^{2}\right) \frac{t^{3 \alpha}}{\Gamma(1+3 \alpha)} \\
& +\cdots
\end{aligned}
$$

Define the residual function for (19)

$$
\begin{aligned}
\operatorname{Res}_{1}(x, t)= & D_{t}^{\alpha} u_{1}-D_{x x} u_{1}-6 u_{1}+12 u_{0} u_{1} \\
& -12 u_{0} D_{t}^{2 \alpha} u_{0}+6 D_{t}^{2 \alpha} u_{0} .
\end{aligned}
$$

Suppose that

$$
\begin{aligned}
u_{1}(x)= & B_{1}(x)+B_{2}(x) \frac{t^{\alpha}}{\Gamma(1+\alpha)}+B_{3}(x) \frac{t^{2 \alpha}}{\Gamma(1+2 \alpha)} \\
& +B_{4}(x) \frac{t^{3 \alpha}}{\Gamma(1+3 \alpha)}+\cdots
\end{aligned}
$$

By the initial condition, the 0 th residual power series approximate solution of $u(x, t)$ can be written as follows:

$$
u_{1}(x, 0)=0=B_{1}(x)
$$

We apply the RPSM to find out $B_{k}(x), k=1,2,3, \ldots, m$, in (19). Thus, we have

$$
\begin{aligned}
u_{1} & =B_{1}+\left(B_{1}^{\prime \prime}+6 B_{1}-12 A_{1} B_{1}+12 A_{1} A_{3}-6 A_{3}\right) \\
& \cdot \frac{t^{\alpha}}{\Gamma(1+\alpha)}+\left(B_{2}^{\prime \prime}+6 B_{2}-12 A_{2} B_{1}-12 A_{1} B_{2}\right. \\
& \left.+12 A_{2} A_{3}+12 A_{1} A_{4}-6 A_{4}\right) \frac{t^{2 \alpha}}{\Gamma(1+2 \alpha)}+\left(B_{3}^{\prime \prime}\right. \\
& +6 B_{3}-12 A_{3} B_{1}-12 A_{1} B_{3}-24 A_{2} B_{2}+12 A_{3}^{2} \\
& \left.+24 A_{2} A_{4}\right) \frac{t^{3 \alpha}}{\Gamma(1+3 \alpha)}+\cdots
\end{aligned}
$$

Define the residual function for (20)

$$
\begin{aligned}
\operatorname{Res}_{2}(x, t)= & D_{t}^{\alpha} u_{2}-D_{x x} u_{2}-6 u_{2}+6 u_{1}^{2}+12 u_{0} u_{2} \\
& -12 u_{0} D_{t}^{2 \alpha} u_{1}-12 u_{1} D_{t}^{2 \alpha} u_{0}+6 D_{t}^{2 \alpha} u_{1} \\
& +6\left(D_{t}^{2 \alpha} u_{0}\right)^{2} .
\end{aligned}
$$

Suppose that

$$
\begin{aligned}
u_{2}(x)= & C_{1}(x)+C_{2}(x) \frac{t^{\alpha}}{\Gamma(1+\alpha)} \\
& +C_{3}(x) \frac{t^{2 \alpha}}{\Gamma(1+2 \alpha)}+C_{4}(x) \frac{t^{3 \alpha}}{\Gamma(1+3 \alpha)} \\
& +\cdots
\end{aligned}
$$

By the initial condition, the $0 t h$ residual power series approximate solution of $u_{2}(x, t)$ can be written as follows:

$$
u_{2}(x, 0)=0=C_{1}(x)
$$

We apply the RPSM to find out $C_{k}(x), k=1,2,3, \ldots, m$ in (20).

Thus, we have

$$
\begin{aligned}
u_{2} & =C_{1}+\left(C_{1}^{\prime \prime}+6 C_{1}-6 B_{1}^{2}-12 A_{1} C_{1}+12 A_{1} B_{3}\right. \\
& \left.+12 A_{3} B_{1}-6 B_{3}-6 A_{3}^{2}\right) \frac{t^{\alpha}}{\Gamma(1+\alpha)}+\left(C_{2}^{\prime \prime}+6 C_{2}\right. \\
& -12 B_{1} B_{2}-12 A_{2} C_{1}-12 A_{1} C_{2}+12 A_{2} B_{3} \\
& \left.+12 A_{1} B_{4}+12 A_{4} B_{1}+12 A_{3} B_{2}-6 B_{4}-12 A_{3} A_{4}\right) \\
& \cdot \frac{t^{2 \alpha}}{\Gamma(1+2 \alpha)}+\left(C_{3}^{\prime \prime}+6 C_{3}-12 B_{3} B_{1}-12 B_{2}^{2}\right. \\
& -24 A_{2} C_{2}-12 A_{3} C_{1}-12 A_{1} C_{3}+24 A_{3} B_{3} \\
& \left.+24 A_{2} B_{4}+24 A_{4} B_{2}-12 A_{4}^{2}\right) \frac{t^{3 \alpha}}{\Gamma(1+3 \alpha)}+\cdots
\end{aligned}
$$


Define the residual function for (11)

$$
\begin{aligned}
\operatorname{Res}_{3}(x, t)= & D_{t}^{\alpha} u_{3}-D_{x x} u_{3}-6 u_{3}+12 u_{0} u_{3} \\
& +12 u_{1} u_{2}-12 u_{0} D_{t}^{2 \alpha} u_{2}-12 u_{1} D_{t}^{2 \alpha} u_{1} \\
& -12 u_{2} D_{t}^{2 \alpha} u_{0}+6 D_{t}^{2 \alpha} u_{2} \\
& +12 D_{t}^{2 \alpha} u_{0} D_{t}^{2 \alpha} u_{1} .
\end{aligned}
$$

Suppose that

$$
\begin{aligned}
u_{3}(x)= & D_{1}(x)+D_{2}(x) \frac{t^{\alpha}}{\Gamma(1+\alpha)} \\
& +D_{3}(x) \frac{t^{2 \alpha}}{\Gamma(1+2 \alpha)}+D_{4}(x) \frac{t^{3 \alpha}}{\Gamma(1+3 \alpha)} \\
& +\cdots
\end{aligned}
$$

By the initial condition, the 0 th residual power series approximate solution of $u_{2}(x, t)$ can be written as follows:

$$
u_{3}(x, 0)=0=D_{1}(x)
$$

We apply the RPSM to find out $D_{k}(x), k=1,2,3, \ldots, m$, in (21).

Thus, we have

$$
\begin{aligned}
u_{3} & =D_{1}+\left(D_{1}^{\prime \prime}+6 D_{1}-12 A_{1} D_{1}-12 B_{1} C_{1}\right. \\
& \left.+12 A_{1} C_{3}+12 A_{3} C_{1}-6 C_{3}-12 A_{3} B_{3}\right) \frac{t^{\alpha}}{\Gamma(1+\alpha)} \\
& +\left(D_{2}^{\prime \prime}+6 D_{2}-12 A_{2} D_{1}-12 A_{1} D_{2}-12 B_{2} C_{1}\right. \\
& -12 B_{1} C_{2}+12 A_{2} C_{3}+12 A_{1} C_{4}+12 B_{2} B_{3} \\
& +12 B_{1} B_{4}+12 A_{4} C_{1}+12 A_{3} C_{2}-6 C_{4}-12 A_{4} B_{3} \\
& \left.-12 A_{3} B_{4}\right) \frac{t^{2 \alpha}}{\Gamma(1+2 \alpha)}+\left(D_{3}^{\prime \prime}+6 D_{3}-12 A_{3} D_{1}\right. \\
& -12 A_{1} D_{3}-24 A_{2} D_{2}-12 B_{3} C_{1}-12 B_{1} C_{3} \\
& -24 B_{2} C_{2}+24 A_{3} C_{3}+24 A_{2} C_{4} 12 B_{3}^{2}+24 B_{2} B_{4} \\
& \left.+24 A_{4} C_{2}-24 A_{4} B_{4}\right) \frac{t^{3 \alpha}}{\Gamma(1+3 \alpha)}+\cdots
\end{aligned}
$$

For $n=3$, we obtain $u_{0}, u_{1}, u_{2}$, and $u_{3}$ as follows:

$$
\begin{aligned}
u_{0}= & A_{1}+\left(A_{1}^{\prime \prime}+6 A_{1}-6 A_{1}^{2}\right) \frac{t^{\alpha}}{\Gamma(1+\alpha)} \\
& +\left(A_{2}^{\prime \prime}+6 A_{2}-12 A_{1} A_{2}\right) \frac{t^{2 \alpha}}{\Gamma(1+2 \alpha)} \\
& +\left(A_{3}^{\prime \prime}+6 A_{3}-12 A_{1} A_{3}-12 A_{2}^{2}\right) \frac{t^{3 \alpha}}{\Gamma(1+3 \alpha)} \\
& +\cdots
\end{aligned}
$$

and

$$
\begin{aligned}
u_{1} & =B_{1}+\left(B_{1}^{\prime \prime}+6 B_{1}-12 A_{1} B_{1}+12 A_{1} A_{4}-6 A_{4}\right) \\
& \cdot \frac{t^{\alpha}}{\Gamma(1+\alpha)}+\left(B_{2}^{\prime \prime}+6 B_{2}-12 A_{2} B_{1}-12 A_{1} B_{2}\right. \\
& \left.+12 A_{2} A_{4}+12 A_{1} A_{4}-6 A_{4}\right) \frac{t^{2 \alpha}}{\Gamma(1+2 \alpha)}+\left(B_{3}^{\prime \prime}\right. \\
& \left.+6 B_{3}-12 A_{3} B_{1}-12 A_{1} B_{3}-24 A_{2} B_{2}+12 A_{3} A_{4}\right) \\
& \cdot \frac{t^{3 \alpha}}{\Gamma(1+3 \alpha)}+\cdots
\end{aligned}
$$

and

$$
\begin{aligned}
u_{2} & =C_{1}+\left(C_{1}^{\prime \prime}+6 C_{1}-6 B_{1}^{2}-12 A_{1} C_{1}+12 A_{1} B_{4}\right. \\
& \left.+12 A_{4} B_{1}-6 B_{4}-6 A_{4}^{2}\right) \frac{t^{\alpha}}{\Gamma(1+\alpha)}+\left(C_{2}^{\prime \prime}+6 C_{2}\right. \\
& -12 B_{1} B_{2}-12 A_{2} C_{1}-12 A_{1} C_{2}+12 A_{2} B_{4} \\
& \left.+12 A_{4} B_{2}\right) \frac{t^{2 \alpha}}{\Gamma(1+2 \alpha)}+\left(C_{3}^{\prime \prime}+6 C_{3}-12 B_{3} B_{1}\right. \\
& -12 B_{2}^{2}-24 A_{2} C_{2}-12 A_{3} C_{1}-12 A_{1} C_{3}+12 A_{3} B_{4} \\
& \left.+12 A_{4} B_{3}\right) \frac{t^{3 \alpha}}{\Gamma(1+3 \alpha)}+\cdots
\end{aligned}
$$

and

$$
\begin{aligned}
u_{3} & =D_{1}+\left(D_{1}^{\prime \prime}+6 D_{1}-12 A_{1} D_{1}-12 B_{1} C_{1}\right. \\
& \left.+12 A_{1} C_{3}+12 A_{3} C_{1}-6 C_{3}-12 A_{3} B_{3}\right) \frac{t^{\alpha}}{\Gamma(1+\alpha)} \\
& +\left(D_{2}^{\prime \prime}+6 D_{2}-12 A_{2} D_{1}-12 A_{1} D_{2}-12 B_{2} C_{1}\right. \\
& -12 B_{1} C_{2}+12 A_{2} C_{3}+12 A_{1} C_{4}+12 B_{2} B_{3} \\
& +12 B_{1} B_{4}+12 A_{4} C_{1}+12 A_{3} C_{2}-6 C_{4}-12 A_{4} B_{3} \\
& \left.-12 A_{3} B_{4}\right) \frac{t^{2 \alpha}}{\Gamma(1+2 \alpha)}+\left(D_{3}^{\prime \prime}+6 D_{3}-12 A_{3} D_{1}\right. \\
& -12 A_{1} D_{3}-24 A_{2} D_{2}-12 B_{3} C_{1}-12 B_{1} C_{3} \\
& -24 B_{2} C_{2}+24 A_{3} C_{3}+24 A_{2} C_{4} 12 B_{3}^{2}+24 B_{2} B_{4} \\
& \left.+24 A_{4} C_{2}-24 A_{4} B_{4}\right) \frac{t^{3 \alpha}}{\Gamma(1+3 \alpha)}+\cdots
\end{aligned}
$$

For $n=4$, we have $u_{0}, u_{1}, u_{2}$, and $u_{3}$ as follows:

$$
\begin{aligned}
u_{0}= & A_{1}+\left(A_{1}^{\prime \prime}+6 A_{1}-6 A_{1}^{2}\right) \frac{t^{\alpha}}{\Gamma(1+\alpha)} \\
& +\left(A_{2}^{\prime \prime}+6 A_{2}-12 A_{1} A_{2}\right) \frac{t^{2 \alpha}}{\Gamma(1+2 \alpha)} \\
& +\left(A_{3}^{\prime \prime}+6 A_{3}-12 A_{1} A_{3}-12 A_{2}^{2}\right) \frac{t^{3 \alpha}}{\Gamma(1+3 \alpha)} \\
& +\cdots
\end{aligned}
$$


and

$$
\begin{aligned}
u_{1}= & B_{1}+\left(B_{1}^{\prime \prime}+6 B_{1}-12 A_{1} B_{1}\right) \frac{t^{\alpha}}{\Gamma(1+\alpha)} \\
& +\left(B_{2}^{\prime \prime}+6 B_{2}-12 A_{2} B_{1}-12 A_{1} B_{2}\right) \frac{t^{2 \alpha}}{\Gamma(1+2 \alpha)} \\
& +\left(B_{3}^{\prime \prime}+6 B_{3}-12 A_{3} B_{1}-12 A_{1} B_{3}-24 A_{2} B_{2}\right) \\
& \cdot \frac{t^{3 \alpha}}{\Gamma(1+3 \alpha)}+\cdots
\end{aligned}
$$

and

$$
\begin{aligned}
u_{2} & =C_{1}+\left(C_{1}^{\prime \prime}+6 C_{1}-6 B_{1}^{2}-12 A_{1} C_{1}\right) \frac{t^{\alpha}}{\Gamma(1+\alpha)} \\
& +\left(C_{2}^{\prime \prime}+6 C_{2}-12 B_{1} B_{2}-12 A_{2} C_{1}-12 A_{1} C_{2}\right) \\
& \cdot \frac{t^{2 \alpha}}{\Gamma(1+2 \alpha)}+\left(C_{3}^{\prime \prime}+6 C_{3}-12 B_{3} B_{1}-12 B_{2}^{2}\right. \\
& \left.-24 A_{2} C_{2}-12 A_{3} C_{1}-12 A_{1} C_{3}\right) \frac{t^{3 \alpha}}{\Gamma(1+3 \alpha)}+\cdots
\end{aligned}
$$

and

$$
\begin{aligned}
u_{3} & =D_{1}+\left(D_{1}^{\prime \prime}+6 D_{1}-12 A_{1} D_{1}-12 B_{1} C_{1}\right) \\
& \cdot \frac{t^{\alpha}}{\Gamma(1+\alpha)}+\left(D_{2}^{\prime \prime}+6 D_{2}-12 A_{2} D_{1}-12 A_{1} D_{2}\right. \\
& \left.-12 B_{2} C_{1}-12 B_{1} C_{2}\right) \frac{t^{2 \alpha}}{\Gamma(1+2 \alpha)}+\left(D_{3}^{\prime \prime}+6 D_{3}\right. \\
& -12 A_{3} D_{1}-12 A_{1} D_{3}-24 A_{2} D_{2}-12 B_{3} C_{1} \\
& \left.-12 B_{1} C_{3}-24 B_{2} C_{2}\right) \frac{t^{3 \alpha}}{\Gamma(1+3 \alpha)}+\cdots
\end{aligned}
$$

\section{Numerical Results}

Consider the following time-fractional Fisher equation with small delay

$$
\begin{aligned}
D_{t}^{\alpha} u(x, t)= & D_{x x} u(x, t) \\
& +6 u(x, t-\epsilon)(1-u(x, t-\epsilon))
\end{aligned}
$$

subject to initial condition

$$
u(x, 0)=\frac{1}{\left(1+e^{x}\right)^{2}} .
$$

Then, the exact solution of (1) without delay when $\alpha=1$ is given by

$$
u(x, t)=\frac{1}{\left(1+e^{x-5 t}\right)^{2}} .
$$

It is clear from Figures 1 and 2 and Tables 1-2 that the graph of the solution $u(x, t)$ of time-fractional Fisher equation with

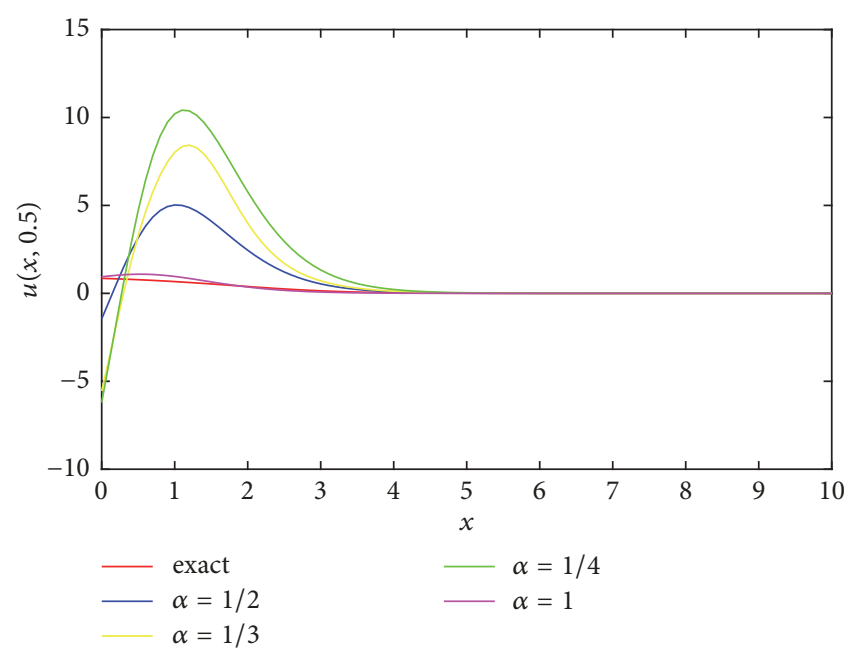

FIGURE 1: $u$ solution for $t=0.5, \epsilon=0.001$, and $0 \leq x \leq 10$.

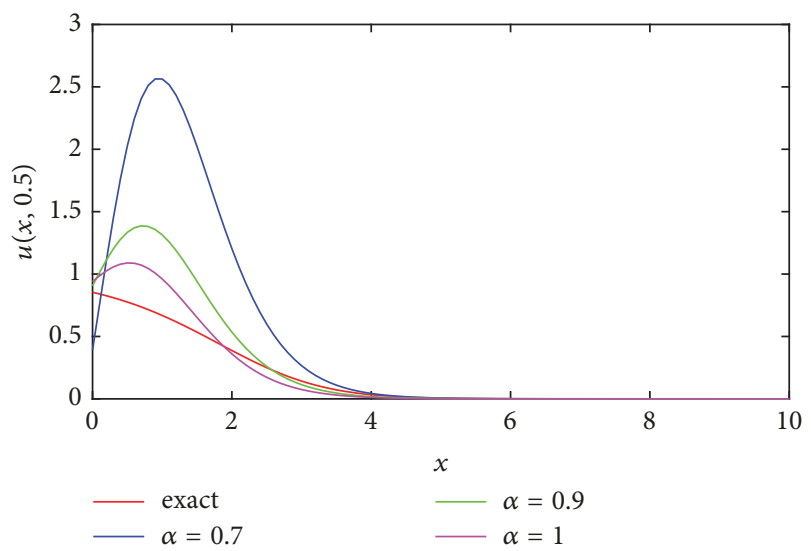

FIGURE 2: $u$ solution for $t=0.5, \epsilon=0.001$, and $0 \leq x \leq 10$.

small delay with respect to space $x$ in some neighborhood of $x=0$ solution acts as a critically damped oscillator for $\alpha<1 / 2$ whereas it acts as an over damped oscillator for $\alpha>1 / 2$. In Figures 3 and 4 , as it is seen from the graph of the solution $u(x, t)$ of time-fractional Fisher equation with small delay with respect to time $t$, the effect of the small time delay is limited for the fractional derivative of order $\alpha<1 / 2$; i.e., the solution $u(x, t)$ of time-fractional Fisher equation with small delay keeps close to the solution $u(x, t)$ of Fisher equation without delay. As a result, it is concluded that the solution $u(x, t)$ is stable under small time delay for $\alpha<1 / 2$. On the other hand, the effect of the small time delay increases as time increases for $\alpha>1 / 2$; i.e., the solution $u(x, t)$ of timefractional Fisher equation with small delay gets away from the solution $u(x, t)$ of Fisher equation without delay. As a result, it is concluded that the solution $u(x, t)$ is unstable under small time delay for $\alpha>1 / 2$.

\section{Conclusion}

Based on the analysis of the solution $u(x, t)$ of time-fractional Fisher equation with small delay, it is deduced that, at the 
TABLE 1: The RPS solution for several values $x$ and $\alpha$ for $t=0.5$.

\begin{tabular}{lccc}
\hline$x$ & $\alpha=1 / 4$ & $\alpha=1 / 3$ & $\alpha=1 / 2$ \\
\hline 0.1 & -3.95998 & -3.72539 & -0.046675 \\
0.2 & -1.65603 & -1.82531 & 0.52762 \\
0.4 & 2.76181 & 1.75026 & 2.37864 \\
0.6 & 6.42178 & 4.62171 & 3.82190 \\
0.8 & 8.93800 & 6.71422 & 4.70664 \\
1 & 10.21002 & 8.02085 & 5.02524 \\
1.5 & 9.17997 & 7.40754 & 4.10699 \\
2 & 5.77391 & 3.99228 & 2.45076 \\
2.5 & 2.95270 & 1.70703 & 1.21563 \\
3 & 1.32987 & 0.70989 & 0.53796 \\
3.5 & 0.55378 & 0.29586 & 0.22174 \\
4 & 0.21974 & 0.12070 & 0.08746 \\
4.5 & 0.08465 & 0.04780 & 0.03358 \\
5 & 0.03203 & 0.01846 & 0.01268 \\
\hline
\end{tabular}

TABLE 2: The RPS solution for several values $t$ and $\alpha$ for $x=0.5$.

\begin{tabular}{lcccc}
\hline$t$ & $\alpha=0.5$ & $\alpha=0.7$ & $\alpha=0.9$ & $\alpha=1$ \\
\hline 0.1 & -0.41551 & 0.75439 & 1.01269 & 0.98597 \\
0.2 & 0.52501 & 1.11315 & 1.11369 & 1.02597 \\
0.4 & 2.29109 & 1.76224 & 1.27825 & 1.07915 \\
0.6 & 3.70390 & 2.24542 & 1.37108 & 1.08652 \\
0.8 & 4.61711 & 2.51281 & 1.38106 & 1.04491 \\
1 & 5.00312 & 2.56283 & 1.31405 & 0.96068 \\
1.5 & 4.24695 & 2.02110 & 0.94079 & 0.65201 \\
2 & 2.61056 & 1.20375 & 0.53506 & 0.36056 \\
2.5 & 1.32027 & 0.59918 & 0.25991 & 0.17244 \\
3 & 0.59119 & 0.26598 & 0.11381 & 0.07484 \\
3.5 & 0.24539 & 0.10985 & 0.04664 & 0.03051 \\
4 & 0.09719 & 0.04338 & 0.01833 & 0.01195 \\
4.5 & 0.03740 & 0.01667 & 0.00702 & 0.00457 \\
5 & 0.01414 & 0.00629 & 0.00265 & 0.00172 \\
\hline
\end{tabular}

fractional derivative of order $\alpha=1 / 2$, there is a transition of the solution $u(x, t)$ with respect to both variables $x$ and $t$.

\section{Nomenclature}

$\Gamma(x): \quad$ Gamma function

$J^{\alpha} f(x)$ : Riemann-Liouville fractional integral

$D^{\alpha} f(x)$ : Caputo fractional derivative

$D_{t}^{\alpha} f(x)$ : Caputo time-fractional derivative.

\section{Data Availability}

The data in our manuscript is obtained by our calculations.

\section{Disclosure}

The previous version of the manuscript is presented in ICOMAA-2018 under the name of "On the Time-Fractional Fisher Equation with Small Delay".

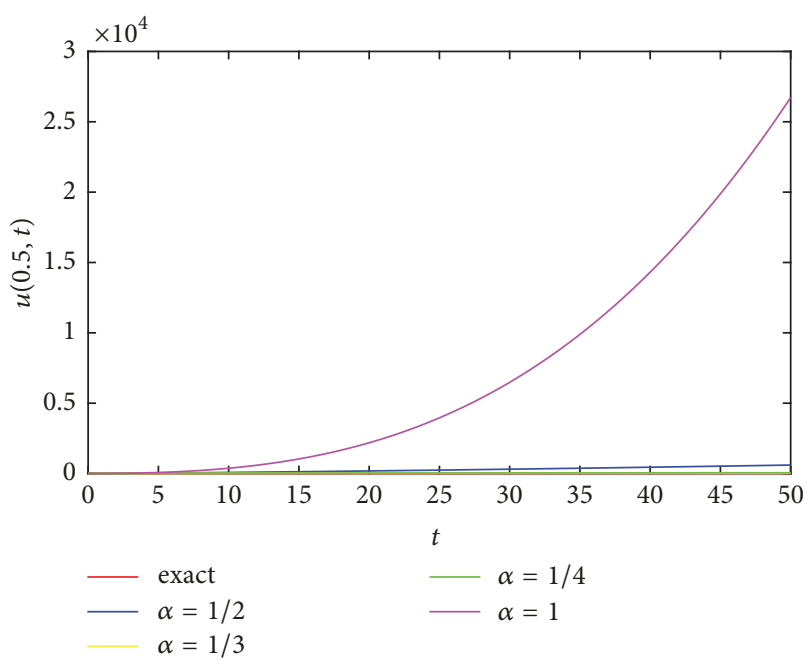

FIGURE 3: $u$ solution for $x=0.5, \epsilon=0.001$, and $0 \leq t \leq 50$.

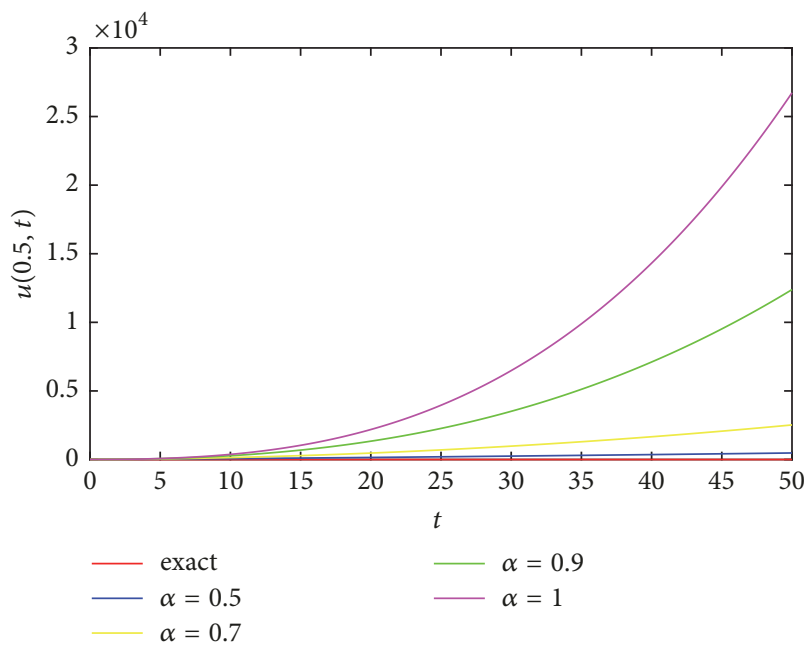

Figure 4: $u$ solution for $x=0.5, \epsilon=0.001$, and $0 \leq t \leq 50$.

\section{Conflicts of Interest}

The authors declare that they have no conflicts of interest.

\section{References}

[1] R. L. Bagley and P. J. Torvik, "A theoretical basis for the application of fractional calilus to visoelasticity," Journal of Rheology, vol. 27, no. 3, pp. 201-210, 1983.

[2] R. L. Bagley and P. J. Torvik, "Fractional calculus-a different approach to the analysis of viscoelastically damped structures," AIAA Journal, vol. 21, no. 5, pp. 741-748, 1983.

[3] R. L. Bagley and P. J. Torvik, "Fractional calculus in the transient analysis of viscoelastically damped structures," AIAA Journal, vol. 23, no. 6, pp. 918-925, 1985.

[4] R. L. Magin, "Fractional calculus in bioengineering," Critical Reviews in Biomedical Engineering, vol. 32, no. 1, pp. 1-377, 2004.

[5] D. A. Robinson, "The use of control systems analysis in the neurophysiology of eye movements.", Annual Review of Neuroscience, vol. 4, pp. 463-503, 1981. 
[6] I. Podlubny, Fractional Differential Equations, vol. 198 of Mathematics in Science and Engineering, Academic Press, San Diego, Calif, USA, 1999.

[7] A. A. Kilbas, H. M. Srivastava, and J. J. Trujillo, Theory and Applications of Fractional Differential Equations, New York, NY, USA, Elsevier, 2006.

[8] V. D. Gejji and A. Babakhani, "Analysis of a system of fractional differential equations," Journal of Mathematical Analysis and Applications, vol. 293, no. 2, pp. 511-522, 2004.

[9] Z. Odibat, "Approximations of fractional integrals and Caputo fractional derivatives," Applied Mathematics and Computation, vol. 178 , no. 2 , pp. 527-533, 2006.

[10] J. Sabatier, O. P. Agrawal, and J. A. Machado, Advance in Fractional Calculus: Theoretical Developments and Applications in Physics and Engineering, Springer, Dordrecht, The Netherlands, 2007.

[11] S. G. Samko, A. A. Kilbas, and O. I. Marichev, Fractional Integrals and Derivatives, Theory and Applications, Gordon and Breach, Yverdon, Switzerland, 1993.

[12] D. Baleanu, K. Diethelm, E. Scalas, and J. J. Trujillo, Fractional Calculus Models and Numerical Methods, vol. 3 of Series on Complexity, Nonlinearity and Chaos, World Scientific, 2012.

[13] O. Abu Arqub, "Series solution of fuzzy differential equations under strongly generalized differentiability," Journal of Advanced Research in Applied Mathematics, vol. 5, no. 1, pp. 3152, 2013.

[14] A. Kumar, S. Kumar, and S.-P. Yan, "Residual power series method for fractional diffusion equations," Fundamenta Informaticae, vol. 151, no. 1-4, pp. 213-230, 2017.

[15] M. Alquran, K. Al-Khaled, and J. Chattopadhyay, "Analytical solutions of fractional population diffusion model: residual power series," Nonlinear Studies. The International Journal, vol. 22, no. 1, pp. 31-39, 2015.

[16] M. Aylin Bayrak and A. Demir, "A new approach for space-time fractional partial differential equations by residual power series method," Applied Mathematics and Computation, vol. 336, pp. 215-230, 2018.

[17] A. El-Ajou, O. Abu Arqub, and M. Al-Smadi, "A general form of the generalized Taylor's formula with some applications," Applied Mathematics and Computation, vol. 256, pp. 851-859, 2015.

[18] O. Abu Arqub, "Application of residual power series method for the solution of time-fractional Schrödinger equations in onedimensional space," Fundamenta Informaticae 2018, In Press.

[19] O. Abu Arqub, Z. Abo-Hammour, R. Al-Badarneh, and S. Momani, "A reliable analytical method for solving higher-order initial value problems," Discrete Dynamics in Nature and Society, vol. 2013, Article ID 673829, 12 pages, 2013.

[20] R. A. Fisher, "The, wave of advance of advantageous genes," Annals of Eugenics, vol. 7, no. 4, pp. 355-369, 1937.

[21] I. C. Sungu and H. Demir, "A New Approach and Solution Technique to Solve Time Fractional Nonlinear Reaction-Diffusion Equations," Mathematical Problems in Engineering, vol. 2015, Article ID 457013, 13 pages, 2015.

[22] S. Z. Rida, A. M. El-Sayed, and A. A. Arafa, "On the solutions of time-fractional reaction-diffusion equations," Communications in Nonlinear Science and Numerical Simulation, vol. 15, no. 12, pp. 3847-3854, 2010.

[23] N. A. Khan, F. Ayaz, L. Jin et al., "On approximate solutions for the time-fractional reaction-diffusion equation of Fisher type," International Journal of Physical Sciences, vol. 6, pp. 2483-2496, 2011.
[24] V. K. Baranwal, R. K. Pandey, M. P. Tripathi, and O. P. Singh, "An analytic algorithm for time fractional nonlinear reactiondiffusion equation based on a new iterative method," Communications in Nonlinear Science and Numerical Simulation, vol. 17, no. 10, pp. 3906-3921, 2012.

[25] M. Merdan, "Solutions of time-fractional reaction-diffusion equation with modified Riemann-Liouville derivative," International Journal of Physical Sciences, vol. 7, no. 15, pp. 2317-2326, 2012.

[26] C. S. Drapaca and S. Sivaloganathan, "A fractional model of continuum mechanics," Journal of Elasticity, vol. 107, no. 2, pp. 105-123, 2012.

[27] W. Sumelka, "Non-local Kirchhoff-Love plates in terms of fractional calculus," Archives of Civil and Mechanical Engineering, vol. 15, no. 1, pp. 231-242, 2015.

[28] Z. Rahimi, G. Rezazadeh, W. Sumelka, and X.-J. Yang, "A study of critical point instability of micro and nano beams under a distributed variable-pressure force in the framework of the inhomogeneous non-linear nonlocal theory," Polish Academy of Sciences. Institute of Fundamental Technological Research. Archives of Mechanics (Archiwum Mechaniki Stosowanej), vol. 69, no. 6, pp. 413-433, 2017.

[29] W. Sumelka and G. Z. Voyiadjis, "A hyperelastic fractional damage material model with memory," International Journal of Solids and Structures, vol. 124, pp. 151-160, 2017.

[30] K. A. Lazopoulos and A. K. Lazopoulos, "Fractional vector calculus and fluid mechanics," Journal of the Mechanical Behavior of Materials, vol. 0, no. 0, 2017.

[31] K. B. Oldham and J. Spanier, The Fractional Calculus, Academic Press, New York, NY, USA, 1974, reprinted in 2006.

[32] K. S. Miller and B. Ross, An Introduction to the Fractional Calculus and Fractional Differential Equations, A Wiley-Interscience Publication, John Wiley \& Sons, New York, NY, USA, 1993.

[33] S. S. Ray, "Analytical solution for the space fractional diffusion equation by two-step Adomian decomposition method," Communications in Nonlinear Science and Numerical Simulation, vol. 14, no. 4, pp. 1295-1306, 2009.

[34] R. Magin, X. Feng, and D. Baleanu, "Solving the fractional order Bloch equation," Concepts in Magnetic Resonance Part A, vol. 34, no. 1, pp. 16-23, 2009.

[35] M. M. Al Qurashi, Z. Korpinar, D. Baleanu, and M. Inc, "A new iterative algorithm on the time-fractional Fisher equation: Residual power series method," Advances in Mechanical Engineering, vol. 9, no. 9, p. 168781401771600, 2017. 


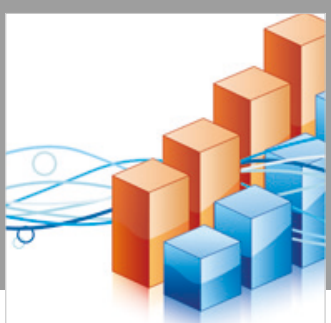

Advances in

Operations Research

\section{-n-m}
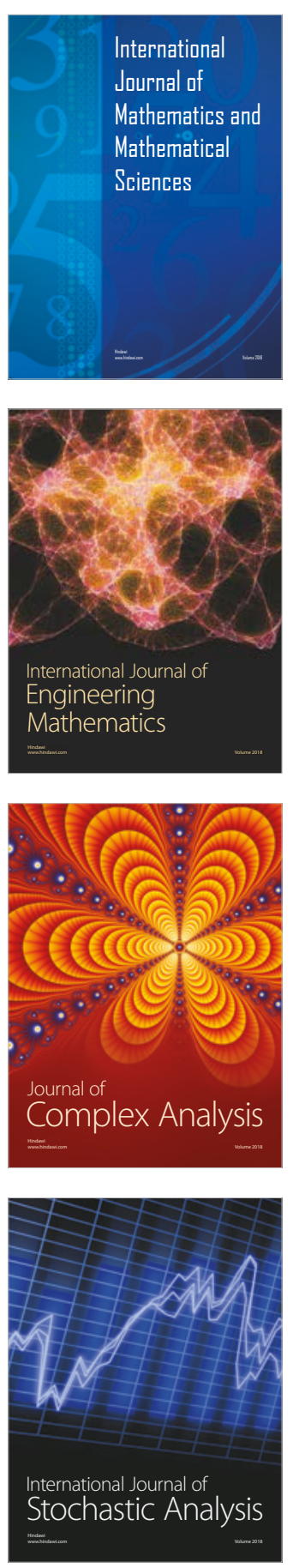
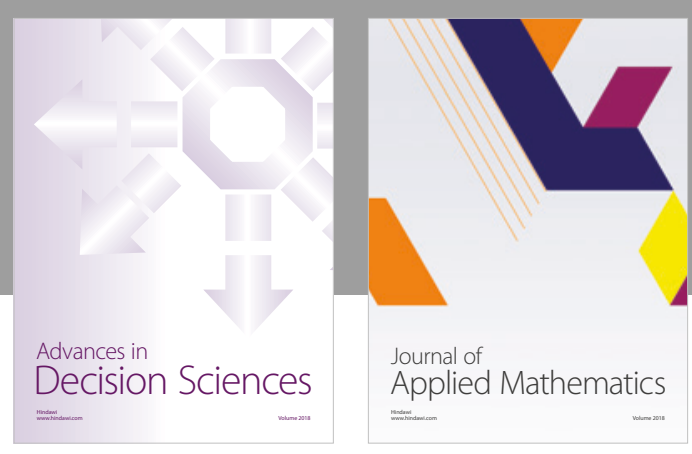

Journal of

Applied Mathematics
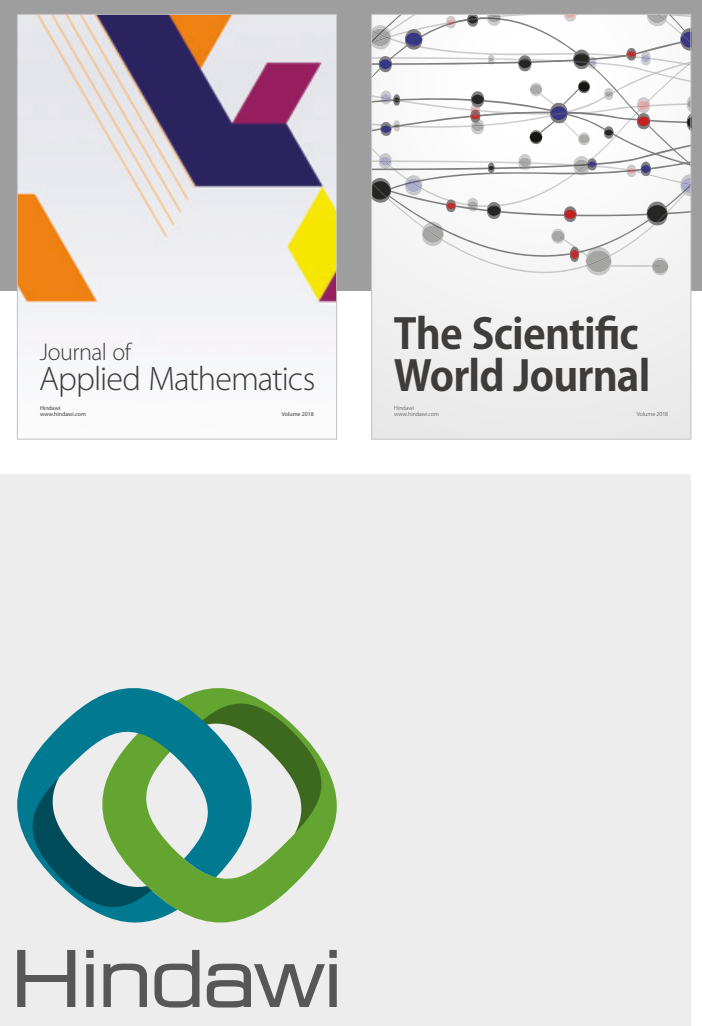

Submit your manuscripts at

www.hindawi.com

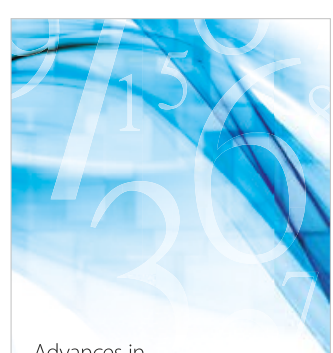

Advances in
Numerical Analysis
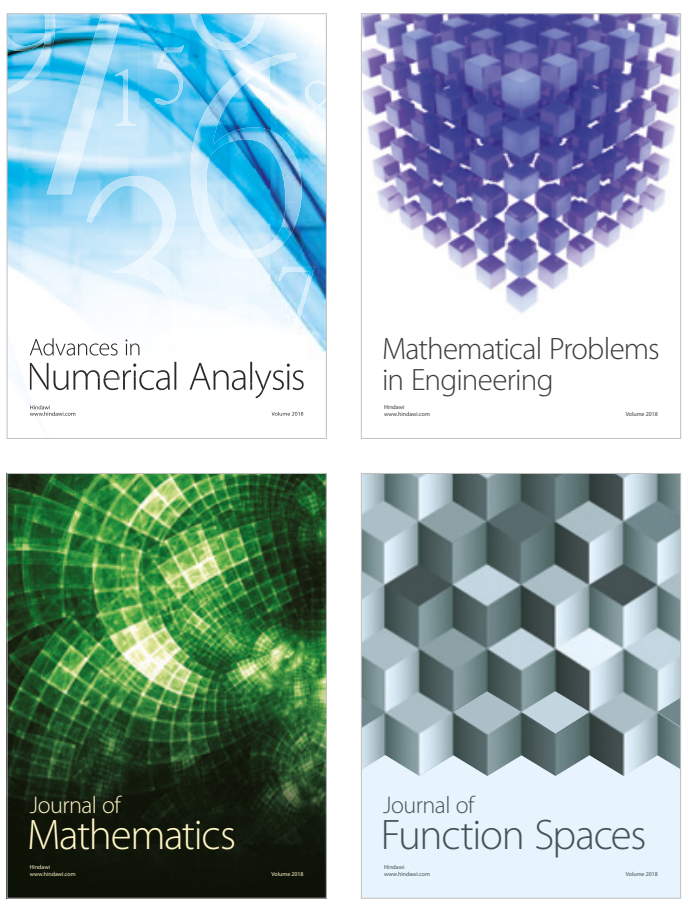

Mathematical Problems in Engineering

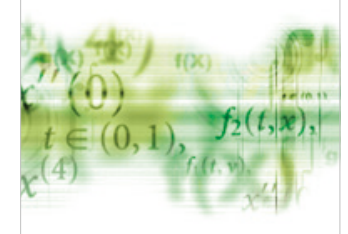

International Journal of

Differential Equations

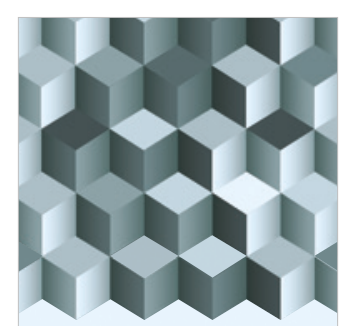

Journal of

Function Spaces

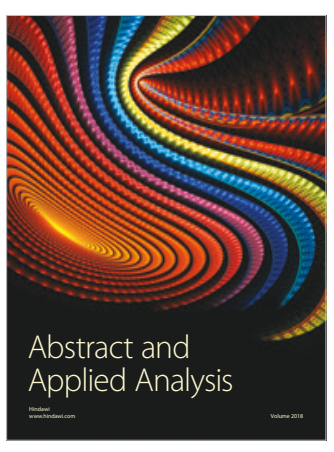

The Scientific

World Journal

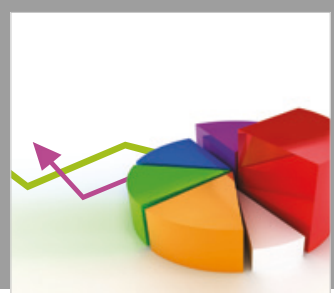

Journal of

Probability and Statistics
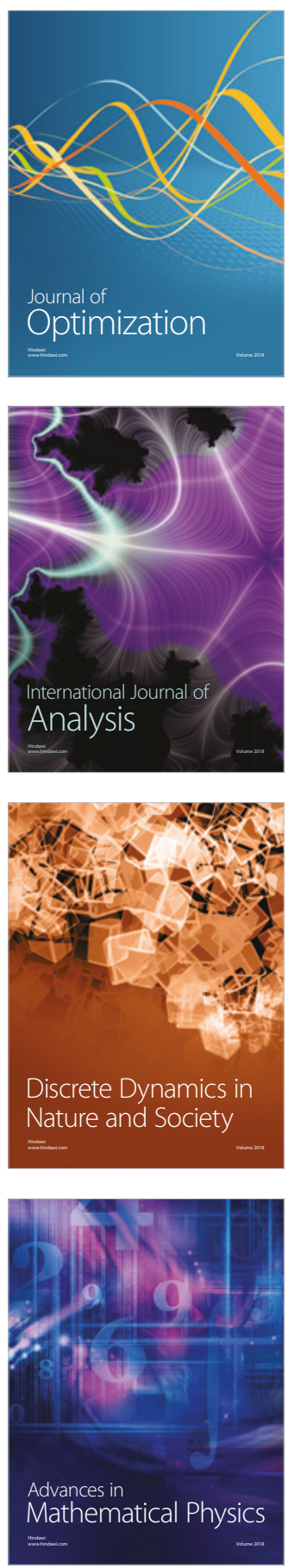